\title{
Review
}

\section{Association of chromosome 6 open reading frame 106 in different cancers}

\author{
Emad Kutbi ${ }^{1}$, Hassan Alsaif ${ }^{2}$, Shahad AlOtaiby ${ }^{3}$, Saeed Baradwan ${ }^{4, *}$ \\ ${ }^{1}$ Department of Biorepository Biomedical Research Administration, Research Center, King Fahad Medical City, 12231 \\ Riyadh, Saudi Arabia, ${ }^{2}$ Faculty of Medicine, Imam Abdulrahman Bin Faisal University, 34212 Dammam, Saudi Arabia, \\ ${ }^{3}$ Department of Scientific Writing Research Services Administration, Research Center, King Fahad Medical City, 12231 \\ Riyadh, Saudi Arabia, ${ }^{4}$ Department of Obstetrics and Gynecology, King Faisal Specialist Hospital and Research Centre, \\ 11564 Jeddah, Saudi Arabia
}

\section{TABLE OF CONTENTS}

\author{
1. Abstract \\ 2. Introduction \\ 3. Role of genes in cancer \\ 4. Chromosome 6 open reading frame 106 (C6orf106) structure \\ 5. Correlation between cancer and C6orf106 \\ 6. C6orf106 interactors \\ 7. C6orf106 animal studies \\ 8. Summary and perspective \\ 9. Author contributions \\ 10. Ethics approval and consent to participate \\ 11. Acknowledgment \\ 12. Funding \\ 13. Conflict of interest \\ 14. References
}

\section{Abstract}

Introduction: Cancer is the leading cause of death globally according to WHO in 2020 . It is initiated by genetic mutations that occur due to numerous factors. The aim of the review: This review provides a clear view of the potential use of chromosome 6 open reading frame 106 (C6orf106) as a biomarker, based on previous studies. Results: Recent studies have investigated the association of C6orf106 with breast cancer and non-small cell lung cancer and showed that silencing C6orf106 leads to inhibition of malignancy in both diseases, as well as showing a positive correlation between C6orf106 expression and malignancy. Other studies demonstrated the interaction of C6orf106 with other malignancy factors that play a role in many cancer types, such as cyclin A2, cyclin B1, N-cadherin, Ecadherin, c-MYC, p120ctn, and vimentin. These factors play a significant role in cellular adhesion and the regulation of the cell cycle. C6orf106 is a potential target for numerous cancers, not only non-small cell lung cancer and breast cancer. In conclusion: understanding the connec- tion of C6orf106 with crucial malignancy factors makes it clear that C6orf106 is a potential therapeutic target and diagnostic biomarker for many disease cancer.

\section{Introduction}

Cancer is a disease that occurs as a result of uncontrolled proliferation and invasion of cells in various sites of the body. The leading cause of cancer is mutations in genes responsible for apoptosis, cell growth, and division, and DNA repair [1]. Genetic changes that contribute to carcinogenesis can be inherited or acquired in genes that play a role in cell-cell adhesion and cell cycle regulation [2]. According to the Global Cancer Observatory, the estimated mortality of cancer in 2018 was 9.5 million, with approximately $25 \%$ of deaths caused by breast and lung cancer [3]. There are several reasons for this high lethality of cancer, including malnutrition as the tumor progresses and inhibition of organ function [4]. Inhibition of organ function occurs after the invasion of cancers cell in the stable tissues of the organ and as angiogenesis occurs with cancer cells con- 
suming more nutrients from the body to survive [4]. One of the main dangers of cancer is its metastatic potential, and it can spread fast making it much harder to be therapeutically controlled and targeted [4].

Current solutions to cure cancer vary based on the type of cancer and its speed (rate to invasion). Generally, patients are administered to a combination of therapy and surgery, chemotherapy, and radiotherapy. Some cancers, such as breast cancer, specifically Human epidermal growth factor receptor 2 (HER2) positive breast cancer can be targeted and treated based on the expression of specific genes or pathways in the cancer cell which decreases the side effects of chemotherapy [5]. The danger of chemotherapy remains a challenge since the drug will be affecting all types of cells including the healthy ones, but in cancers with specific receptors, the target can be narrowed reducing the side effects [6]. Another type of breast cancer is triple-negative breast cancer, which lacks expression of any known cellular markers that can be targeted therapeutically, this makes it much harder to be treated without harming healthy tissues [7]. Another challenge of cancer is an early diagnosis of the malignant tissue, which allows the tumor to be removed as early as possible before metastasis occurs [8].

A rising technique for detecting cancer cells in the body is the screening of cancer biomarkers. A biomarker is a traceable biological indicator such as molecules in the body obtained from blood or tissue fluids, genes, and pathological particles that are indicators of exposure, effect, susceptibility, or clinical disease. Cancer biomarkers can be used at different stages of treatment, including screening, diagnosis, pharmacodynamics, and prediction of recurrence [9]. One example of a famous biomarker is BRCA1 (breast cancer gene), a genetic test of BRCA1 can estimate the risk of developing breast and ovarian cancers in women [10]. The p53 tumor suppressor is another biomarker that is important in tumor suppression. As a transcription factor, p53 primarily suppresses tumors by regulating the transcription of many target genes [11]. Another well-known biomarker is the ERB2 gene in breast cancer, which determines the response to trastuzumab [9]. Another known biomarker which has been reported recently is Kirsten rat sarcoma viral oncogene homolog (KRAS), an oncogene and member of the RAS family is an example of a highly mutated gene in non-small cell lung cancer (NSCLC) patients [12]. A study has shown how KRAS mutation may be a prognostic factor of colorectal cancer patients who undergo lung mastectomy [13].

Cancer biomarkers are showing continuous growth of discovery to take an essential role both therapeutically and diagnostically. The open reading frame (ORF) is a stretch of DNA encoding an uncharacterized protein shown to have potential. Due to the novelty of ORFs, studies on their association with cancer are scarce with the emerging potential of cancer biomarkers. A study by Delgado et al. [14] was able to identify 62 oncogenic
ORFs, one of them is C19orf62, which was found to be a new component of the BRCA1-A complex in breast cancer. Another example is C11or f93, which was found also in the ORF region, which was correlated with colorectal cancer. ORFs show a wide field of novel genetic associations, most importantly in cancer. One of the novel emerging biomarkers is chromosome 6 open reading frame 106 (C6orf106) that recently showed positive signs to be a therapeutic target.

\section{Role of genes in cancer}

Cancer is a genetic disease and there are 2 types of existing cancer genes: the oncogenes, which function as positive growth regulators which are responsible for cellular growth and differentiation, and the tumor suppressor genes, which function as negative growth regulators that are responsible for the genetic integrity, and the mismatch repair (MMR) system which is capable of editing errors in the genetic material during DNA replication [15]. The human MMR system consists of a group of proteins that initiate mismatch repair which if mutated could be responsible for the arousal of cancer, including the core proteins MLH1, MLH3, MSH2, MSH3, and other proteins involved in the DNA MMR pathway such as DNA polymerase delta, RPA, and PCNA $[16,17]$. Thousands of mutations may occur when DNA repair genes are not expressed, which frequently occurs in cancer [18].

\section{Chromosome 6 open reading frame 106 (C6orf106) structure}

C6orf106 arising as a novel marker as its association was proven by its knockdown in several cancers, including NSCLC and breast cancer [7, 19]. C6orf106 was found to have an association with molecules responsible for cell cycle regulators such as cyclins and cellular adhesion molecules [19]. In this review, we summarize the recent insights regarding C6orf106 and we share the current knowledge about C6orf106 and its possible implications in cancer.

\subsection{C6orf106 gene location}

C6orf106 recently named ILRUN (Inflammation And Lipid Regulator With UBA-Like And NBR1-Like Domains) is found at chromosome 6 at the exact cytoband location p21.31 (Fig. 1, Ref. [20]) [7]. Approximately 6\% of the human genome is found on chromosome 6 and the terminal sequence includes $166,880,988$ base pairs which is the largest chromosome sequence [21]. The previous hgnc symbol for the ILRUN gene was C6orf102. The protein coding gene ILRUN functions as a negative regulator of innate antiviral response. It acts as a suppressor of the innate antiviral response. blocks IRF3 (Interferon Regulatory Factor 3) -dependent cytokine production such as IFN (Human 


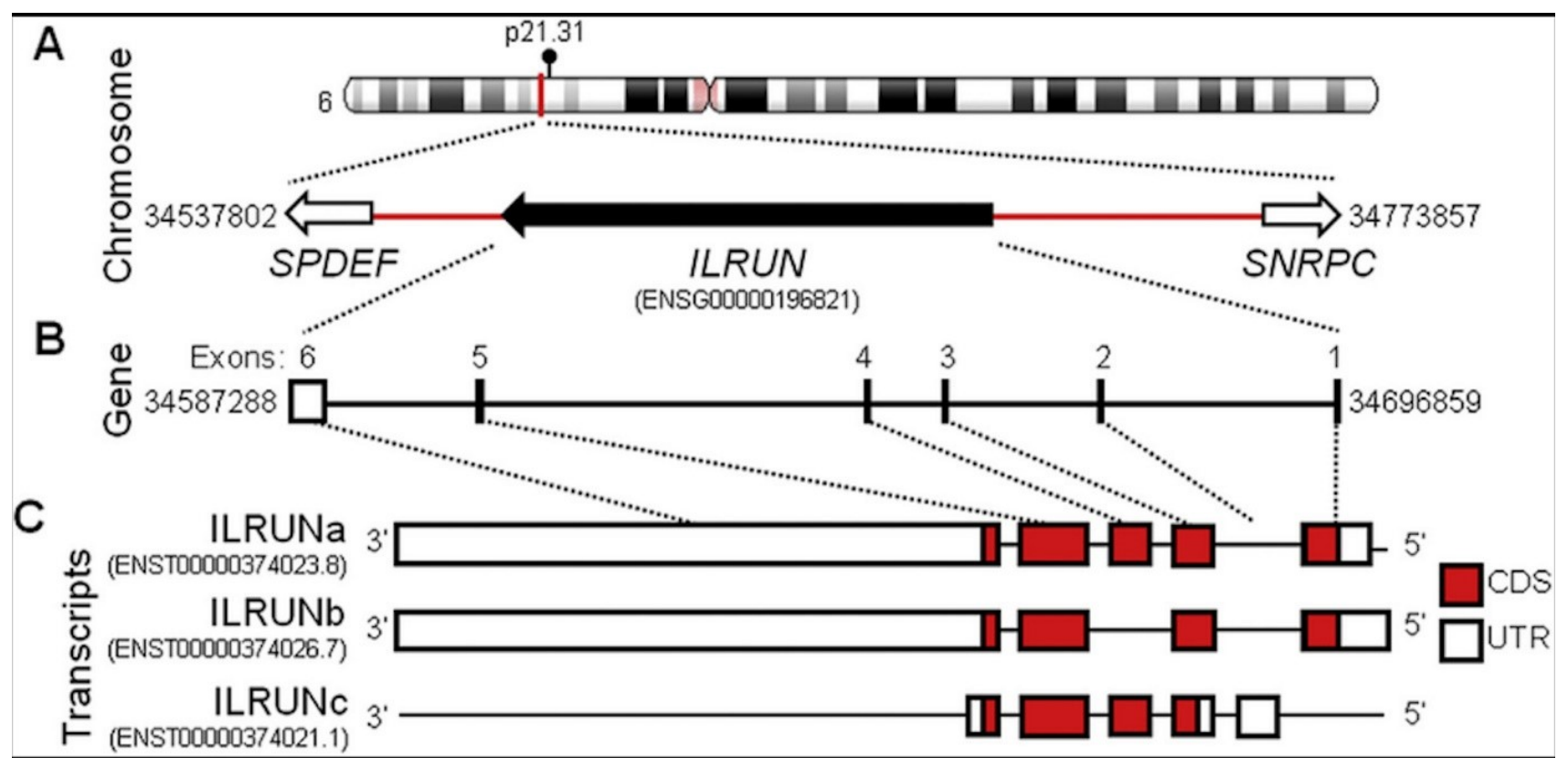

Fig. 1. C6orf106 gene location in the human chromosome. The figure shows the human ILRUN locus is organized genomically. (A) Schematic of the human ILRUN gene, which is localized to chromosome 6; the chromosome image is from the Genome Decoration Page (NCBI). The direction of gene transcription is indicated by arrows. (B) The human ILRUN gene's intron-exon organization. (C) The ILRUN gene produces three transcripts; ILRUNa lacks exon 2 and exon 1 is $5^{\prime} 0$ terminally truncated [20].

type I interferons)- $\alpha$, IFN- $\beta$ and TNF (Tumor necrosis factor). Interacts with IRF3 and prevents it from recruiting to type I IFN promoter sequences, as well as lowering nuclear levels of the coactivators EP300 (p300) and CREBBP. The protein details for the ILRUN gene are unknown, however a suggested name is protein ILRUN, with the accession number Q9H6K1 [22]. Additional previous studies have shown that genes in chromosome 6 directly relate to cancer, schizophrenia, autoimmunity, and many other diseases [21]. A strong correlation was reported between 6p21.31 position and chronic lymphocytic leukemia, this observation strongly supports the association of C6orf106 and cancer [23]. In a study conducted to compare the molecular features of basal-like tumors with the BRCA1 gene mutated with basal-like tumors wild type, 6p21.31 was found amplified in basal-like BRCA1-mutated tumors [24]. Amplification of the gene can ultimately lead to overexpression of the protein it encodes. So, if the protein encoded by the amplified gene is a growth factor receptor that activates the cell cycle for replication, this will ultimately lead to increased proliferation of the cells. Basal-like breast cancer, a subtype that also lacks (PR (Progesterone hormone receptor), ER (estrogen hormone receptor), and HER2 (human epidermal growth factor receptor 2) overexpression is characterized by the overexpression of basal markers such as cytokeratins 5 , 6 , and 17 [25]. This indication can be used to be able to determine novel biomarkers of basal-like breast cancer since it lacks the well-known breast cancer biomarkers.

\subsection{C6orf106 expression}

The expression of C6orf106 mRNA and protein in various cell lines, tissues, and pathological samples have been assessed by the elevated level of expression in certain types of tissues may indicate the importance of C6orf106 as a therapeutic target.

At the cellular level C6orf106 was detected in nuclear speckles of the cell and there are five cell lines found to have the highest expression of C6orf106 mRNA. These cell lines are HDLM-2 (Hodgkin lymphoma cells originating from pleural effusion), HAP1 (myeloid leukemia cells), U-266/84 (lymphoid cancer cells originating from the peripheral blood), SiHa (female reproductive system cancer cells arising from the cervix), and K-562 (myeloid cancer cells originating from the peripheral blood) [26]. The elevated level of expression of C6orf106 mRNA indicates how C6orf106 can be used as a therapeutic target.

C6orf106 was also found to be expressed at the tissue level in different organs from different systems. First, several tissue types have shown some level of expression of C6orf16 mRNA, but it was highest in the skeletal muscle $[27,28]$. On the other hand, Protein expression is high in the cerebral cortex, adrenal gland, nasopharynx, bronchus, gallbladder, salivary gland, small intestine, colon, urinary bladder, seminal vesicle, and breast [28]. One experimental study performed a knockdown of CG5445 (the Drosophila ortholog of C6orf106) and showed accumulation of ubiquitinated proteins, which, in humans, leads to a neurodegenerative disease called amyotrophic lateral sclerosis (ALS) 
$[28,29]$. Further experimental studies can be conducted in vivo to help to determine the lethality of C6orf106 knockdown. This will provide a better understanding of both the benefit of targeting C6orf106 therapeutically and the function of the protein encoded by C6orf106.

\section{Correlation between cancer and C6orf106}

The main function of C6orf106 in cancer, and other diseases has not been well understood yet, however, recent studies emphasized that there is a definite relation between the expression level of C6orf106 and triple-negative breast cancer [7] and pancreatic cancer [30]. A recent study also indicated that C6orf106 is an inhibitor of the antiviral response by regulating interferon regulator factor 3dependent cytokine synthesis [31]. In the below two paragraphs we summarize the recent knowledge about C6orf106 breast cancer and pancreatic cancer.

\subsection{C6orf106 and breast cancer}

Previous experimental studies have been conducted to discover the relationship between C6orf106 expression and cancer progression. One study showed that C6orf106 promotes the malignant progression of breast cancer, specifically triple-negative breast cancer. The methodology was based on patient cells, 78 samples of healthy breast tissue, 59 samples of breast ductal carcinoma in situ, 97 samples of triple-negative breast cancer (TNBC) (a type of breast cancer cells that lack PR, ER, and HER2 expression, and 89 samples of triple-positive breast cancer cells. The C6orf106 expression was examined in the reported cells and they found C6orf106 is expressed in 97\% of breast ductal carcinoma in situ samples, $79.57 \%$ of invasive breast cancer samples, and 3.85\% of normal breast duct glandular epithelial samples. Moreover, there was a positive correlation between the level of expression of C6orf106 and TNM stage (TNM staging well-known classification system with $\mathrm{T}$ standing for tumor size, $\mathrm{N}$ for nearby lymph node metastasis, and $\mathrm{M}$ for distant metastasis) $(p=0.001)$ and lymph node metastasis $(p=0.018)$; however, there was no correlation with age or expression of ER, PR, or HER2. Studies have also examined the role of C6orf106 in MCF10A (normal human mammary cell line), MCF-7 (breast cancer cell line), MDA-MB-231 (breast cancer cell line), and BT-549 (breast cancer cell line) cells. Knockdown of C6orf16 in MDA-MB-231 and BT-549 decreased the cancer cell's ability to invade. Moreover, these cells also had reduced levels of cyclin A2, cyclin B1, c-MYC, and Ncadherin, whereas an elevated level of E-cadherin was observed [7].

Another experimental study on NSCLC has proven that C6orf106 enhances the invasion of NSCLC cells by upregulating vimentin (A type 3 intermediate filament) and downregulating E-cadherin (A tumor suppressor gene with cell to cell adhesion function) and p120ctn (cell to cell adhesion molecule with signal transduction role). In this study, tumor biopsies were obtained from 124 patients, 67 with adenocarcinoma, and 57 with lung squamous cell carcinoma. After evaluation of lymph node metastasis staging and level of differentiation, western blotting was performed on 16 biopsies, of which 13 had increased C6orf106 expression levels, compared to adjacent non-cancerous tissues. Immunohistochemistry was also performed on NSCLC tissues from patients and compared with normal non-cancerous tissues from the same patient and C6orf106 expression was either negative or weak in normal bronchial epithelial and highly expressed in lung cancer tissues. The study also performed gene silencing of C6orf106 in HBE (Bronchial epithelial with cystic fibrosis), A549 (adenocarcinomic human alveolar basal epithelial), H460, H292 (mucoepidermoid pulmonary carcinoma), and LK2 (squamous cell carcinoma) cell lines. Data of this study also showed increased levels of C6orf106 mRNA in all cell lines except HBE. Matrigel invasion assays were also performed, showing a significant increase in cell invasion of overexpressed C6orf106 cells, and a decrease in the invasion when C6orf106 is silenced. Clinical studies have found a correlation between C6orf106 overexpression and clinicopathological features in NSCLC. A positive correlation was found with TNM stage differentiation (and lymph node metastasis C6orf106 overexpression also enhanced invasion and knockdown decreased invasion also C6orf106 overexpression showed an upregulation of vimentin and downregulation of E-cadherin and p120ctn [7].

\subsection{C6orf106 and pancreatic cancer}

Similar results were also shown in an experimental study examining the expression of C6orf106 in pancreatic cancer. C6orf106 was overexpressed in 91 samples of pancreatic tumor tissue. There was also a clear correlation between the advanced TNM stage and lymph node metastasis. Moreover, proliferation and colony formation were enhanced after C6orf106 overexpression. This study also identified C6orf106 as an activator of ERK (The extracellular-signal-regulated kinase)-P90RSK signaling, which conducts communications between the cellular membrane and the nucleus to regulate transcription [32].

According to the Human Protein Atlas, pancreatic, ovarian, and breast cancer patients showed the highest percentage of expression of C6orf106 protein [28]. All these studies confirm the correlation of C6orf106 with breast cancer and pancreatic cancer, although they do not provide a clear association with cancer and malignancy in general.

\section{C6orf106 interactors}

Cellular functions and structure remain stable as long as the responsible proteins are stable, but once one protein is abnormally functioning, it can lead to an unstable environment of cells, which can ultimately lead to cancer. 
Previous studies have identified C6orf106 as an interactor with several factors that were already recognized to affect carcinogenesis. In this section, we classified C6orf106 interactors as signaling pathway factors, cellular receptors, and adhesion molecules. The purpose of this section is to provide an overview of recently discovered interactors and how each of these interactors is affecting carcinogenesis and how C6orf106 is interacting with it.

\subsection{APP (Amyloid-beta precursor protein)}

APP (Amyloid-beta precursor protein) has been found in a reconstituted complex with C6orf106 [33]. Increased APP expression has a significant correlation with cancer cell proliferation in various types of cancer, such as pancreatic, lung, colon, and breast [34-36]. Moreover, APP and APLP2 (an APP family member) are related to increased tumor cell proliferation, migration, and invasion. Also, APP family members have essential functions in many cancers, such as cancers of the prostate, breast, colon, thyroid, lung, nasopharynx, and gastrointestinal tract [37].

\subsection{ELAVL1}

ELAVL1 a member of the ELAVL family of RNAbinding proteins (RBPs), has been shown to interact with C6orf106 by affinity capture-RNA [38]. Previous studies have focused on this protein due to its ability in regulating mRNA stability [39]. Several studies demonstrated the role of ELAVL1 in cancer progression and metastasis in several types of cancer including colorectal, breast, and pancreatic cancer [40]. Previous clinical data showed that overexpression of ELAVL 1 was remarkably related to clinicopathological features, advanced stage, positive lymph nodes, and reduced survival in cancer patients [41]. A clear view of the interaction of ELAVL1 and C6orf106 remains unknown.

\subsection{FBXO25}

Another interactor is FBXO25, a member of the F-box family of proteins that play a crucial role in tumorigenesis [42]. Even though FBXO25 is highly expressed in prostate cancer, a study clarified the importance of FBXO25 in the proliferation, migration, and invasion of NSCLC [28, 42]. Also, it has been indicated that reexpression of FBXO25 in FBXO25-knockout Mantle Cell Lymphoma (MCL) cells enhances cell death, demonstrating its function as a tumor suppressor [43]. Moreover, they found that the knockdown of FBXO25 significantly increased lymphoma development, which overall broadens the idea of using C6orf106 as a therapeutic target because of its biochemical interaction with FBXO25 [43].

\subsection{Heat shock proteins HSPA}

HSPA8 and HSPBP1, two genes encoding heat shock proteins, have been shown to interact with various proteins, including C6orf106 using mass spectrometric methods [44]. HSPs are located both intracellularly and extracellularly, which allows them to protect the cell against environmental stress and act as potent stimulators of the immune response [45]. In a study conducted on endometrial carcinoma, siRNA knockdown of HSPA8 reduced cell proliferation, stimulated cell apoptosis, and suppressed cell growth. HSPA8 plays a significant role in endometrial carcinoma, and it has been used as a candidate biomarker in the early diagnosis and therapy of Stage I endometrial carcinoma [46]. Another study on HSPBP1 showed that tumor cells that have a high HSPBP1/HSP70 molar ratio are more sensitive to anticancer drugs than those with a low rate [47]. According to Uhlen et al. [48], HSPA8, and HSPBP1 are overexpressed in colorectal, breast, prostate, and lung cancers. HSPBP1 has not been identified as a prognostic factor in any human cancer; in contrast, HSPA8 is a prognostic factor in colorectal, liver, and renal cancers. The significance of the interaction with C6orf106 remains unknown.

\subsection{MAP3K1}

As mentioned earlier that a single molecule can affect the entire signaling pathway of a cellular function. The MAPK signaling pathway is activated by C6orf106 in pancreatic cancer. This leads to the upregulation of several factors that enhance tumor invasion [30]. MAP3K1 is a member of a signaling cascade pathway that regulates the expression of cell cycle and differentiation proteins and has been proven to have ubiquitination activity with C6orf106, However the interaction is still not validated yet $[49,50]$. Several studies have shown that the expression of the inactive form of MAP3K1 or mutating its cleavage site will lead to the inhibition of MAP3K1-mediated apoptosis [51, 52]. MAP3K1 also plays a role in the migration of cancer cells and silencing MAP3K1 results in decreasing metastasis [53, 54].

\subsection{SMURF1 (SMAD specific E3 ubiquitin-protein ligase 1)}

SMURF1 is another gene proven to interact with C6orf106 for ubiquitination and found highly expressed in pancreatic, colorectal, and breast cancer tissues [48, 55]. SMURF1 plays a role in protein ubiquitination, which can regulate the cell cycle and tumor formation and progression. SMURF1 encodes an E3 ubiquitin ligase that promotes the ubiquitination of targeted molecules [56]. This specificity of the E3 ligase and the proven interaction of SMURF1 with C6orf106 suggests that SMURF1 is used to degrade C6orf106 protein, which may cause inhibition of metastasis, yet the nature of C6orf106 and SMURF1 remains undetermined.

\subsection{TRIM25 (tripartite motif containing 25)}

TRIM25 is a member of the tripartite motif family, which are also E3 ubiquitin ligases [57, 58]. TRIM25 ubiquitinates bind to estrogen receptor alpha in the presence of estrogen, and also ubiquitinates its coactivators, leading to their degradation [57]. A previous study has also shown a role for TRIM25 in lung cancer; siRNA knockdown of 
TRIM25 caused inhibition of cancer cell migration and proliferation [59]. Mutant P53, a well-known carcinogenesis factor, was found inhibited by TRIM25 [60]. A study has been done to determine the targets of TRIM25 using affinity capture RNA process identified C6orf106 as one of the targets [61]. The significance in the therapeutic or diagnostic scope remains unknown.

\subsection{RPSA (ribosomal protein SA)}

RPSA is a multifunctional protein that acts as a cell surface receptor for laminin. There is a correlation between the expression of RPSA and metastasis in several cancers, including prostate, lung, ovary, and breast cancers [62]. RPSA can activate the MAPK signaling cascade, which enhances the invasion and progression of cancer [63]. A study was done to establish the human liver protein interaction network, demonstrated a physical association between C6orf106 and RPSA using a two-hybrid experiment [64]. Further studies should consider whether the malignancy rate increases when C6orf106 expression increases alongside RSPA. If metastasis was found to be growing when C6orf106 binds to RSPA, then we should consider targeting the binding site of RSPA, which will inhibit the binding of these two proteins, resulting in inhibition of metastasis.

\subsection{Exportin 1(XPO1)}

The nuclear transport protein that exports several proteins from the nucleus to the cytoplasm [65]. The previous study proved the interaction of XPO1 and C6orf106 using mass spectrometric methods, However the interaction is still not validated yet [66]. Further studies should demonstrate if C6orf106 expression is positively correlated with XPO1 and malignancy, and how targeting XPO1 as a therapeutic target to inhibit the transport of C6orf106 will result in inhibition of cancer malignancy.

\subsection{Cyclins \\ 6.10.1 Cyclin A2}

Cyclin A2 is a cell cycle regulator that is increased during the $\mathrm{S}$ phase of the cell cycle [67]. A previous study conducted a knockout experiment of CCNA2, the gene encoding cyclin A2, in mice treated with 7,12dimethylbenz(a)anthracene. The results showed that mice with knocked-out CCNA2 or Cyclin A2 showed an increase in tumor incidence, proving that cyclin A2 insufficiency enhances tumor progression [68]. Another study demonstrated C6orf106 as a novel biomarker for breast cancer also showed that silencing C6orf106 reduced the expression of cyclin A2 (Table 1, Ref. [5, 12, 60]) [7]. This was the first study to suggest that C6orf106 plays a role in tumor progression.
Table 1. Summary of the impact of overexpressing C6orf106 on different regulators based on previous experiments.

\begin{tabular}{lccc}
\hline Protein & Impact of C6orf106 overexpression & Cell lines & Reference \\
\hline Cyclin A2 & Downregulated & MDA-MB-231 & {$[5]$} \\
& & BT-549 & \\
Cyclin B1 & Downregulated & MDA-MB-231 & {$[5]$} \\
& & BT-549 & \\
C-MYC & Downregulated & MDA-MB-231 & {$[5]$} \\
& & BT-549 & \\
Vimentin & Upregulated & A549 & {$[12]$} \\
& & SPC & \\
N-cadherin & Downregulated & MDA-MB-231 & {$[5]$} \\
& & BT-549 & \\
& & MDA-MB-231 & {$[5]$} \\
E-cadherin & Upregulated & BT-549 & {$[12]$} \\
& & A549 & {$[60]$} \\
& & SPC & \\
P120ctn & Downregulated & A549 & {$[12]$} \\
& & SPC & \\
\hline
\end{tabular}

\subsubsection{Cyclin B1}

Cyclin B1, encoded by CCNB1, is responsible for the transition from the $\mathrm{G} 2$ phase to the $\mathrm{M}$ phase $[69,70]$. Overexpression of CCNB1 has been found in cancer cells, especially in the prostate $[69,71]$. Silencing CCNB1 in KB3-1 (endocervical adenocarcinoma), SK-N-MC (neuroepithlioma), MCF7, and HL-60 (human leukemia cell lines) cells caused a decrease in cell growth only in MCF7 cells, but not in any of the other lines [69]. In another study, the silencing of C6orf106 caused downregulation of cyclin B1G (Table 1, Ref. [5, 12, 60]) [7]. That C6orf106 affects multiple growth factors underlines the need to carefully develop a narrowly targeted gene silencing technique to inhibit other factors that contribute to metastasis.

\subsubsection{Cyclin D1 and cyclin E1}

Cyclin D1 and E1 are also upregulated in pancreatic cancer tissue as a result of activation of the MAPK/ERK signaling pathway. An experimental study showed this relationship by silencing C6orf106 and comparing the expression of cyclin D1 and E1 in the Panc1 and Capan2 cell lines (pancreatic cancer cell lines). Results have shown Panc1, and Capan2 cells with overexpressed C6orf106 had upregulated ERK signaling pathway, which regulates Cyclin D1 and E1 [30].

\subsection{1 Мyc}

Myc is a family of proto-oncogenes activated in more than half of human cancers [72]. Myc was found to have a role in apoptosis signaling; however, this does not apply in every case. Some cancers are reversed under the suppression of Myc, meaning that Myc could be beneficial in certain cancer situations and highly destructive in others [72]. C-Myc is another gene that is downregulated by the silencing of C6orf106 [7]. C-Myc is considered a master 
regulator that impacts both cellular metabolism and cellular growth [73]. This suggests we need further knowledge about the effect of C6orf106 on Myc in different cancers. Myc is a clear example that encourages researchers to investigate further- the impact of silencing C6orf106 in various cancers, as not all cancers show the same effect.

\subsection{Cadherin}

\subsubsection{CDH1}

CDH1, also known as E-cadherin, is an adhesion molecule in epithelial cells and also known to be a tumor suppressor in carcinomas. It has been shown to have tumorpromoting activity in inflammatory breast, ovarian, and squamous cell carcinoma. E-cadherin was found upregulated when C6orf106 was silenced in NSCLC [19]. This indicates that C6orf106 increases the malignancy of NSCLC by reducing the levels of E-cadherin, which has shown similar results in breast cancer studies [7]. This shows the effectiveness of targeting C6orf106 in carcinomas in general. Snail, a protein that inhibits E-cadherin transcription by binding to its promoter, is upregulated in pancreatic cancer tissue, which also enhances tumor invasion (Table 1, Ref. [5, 12, 60]) [30].

\subsubsection{CDH2}

$\mathrm{CDH} 2$ is another member of the cadherin family is also known as neuronal cadherin ( $\mathrm{N}$-cadherin) that is affected by targeting C6orf106 [7, 19]. CDH2 has a dual role in cancer progression, as it can act as a tumor suppressor in neuroblastoma and as a tumor promoter in melanoma and other carcinomas [74]. The impact of c6orf106 on $\mathrm{N}$-cadherin can be further studied to determine the role of C6orf106 in cancer progression.

\subsection{Vimentin}

Vimentin is a type III intermediate filament that has an expression correlation with several types of cancers, including prostate, gastric, breast, and NSCLC [75]. In cancer, it influences many steps of the epithelial-tomesenchymal transition (EMT) of tumor cells in both a direct and an indirect way [76]. EMT is considered the first step in the metastatic cascade, followed by penetration of the basement membrane, separation from the original tumor, invasion into different tissue, and formation of another tumor at a different site [77]. Since Vimentin is found upregulated by c6orf106, we can further understand how silencing c6orf106 will inhibit the rate of metastasis as Vimentin impacts a key metastasis transition.

\subsection{P120ctn}

P120ctn plays a significant role in the stability of cadherins, it binds to the cytoplasmic tail of cadherins and regulates the stability of cadherin production on the cell surface [78]. The role of p120ctn in cancer is major, and it affects cadherins, MAPK signaling, and the Kaiso transcription factor, among other factors [78]. P120ctn was found downregulated after C6orf106 gene silencing in NSCLC (Table 1, Ref. [5, 12, 60]) [18]. This can explain the downregulation of E-cadherin and the crosstalk between C6orf106 and the MAPK signaling pathway.

\section{C6orf106 animal studies}

The ILRUN knock-out mice had significantly lower plasma cholesterol levels resulting from reduced liver lipoprotein production. This result identified ILRUN as a new lipid metabolism regulator that increases the synthesis of hepatic lipoproteins. Also, the study findings further show that ILRUN may be the incidental gene responsible for the observed genetic correlations with plasma lipids at 6 p21 in humans [79].

\section{Summary and perspective}

This review demonstrated the network of tumor progression regulators that are affected by targeting C6orf106. The literature review of several articles about C6orf106 shows how targeting C6orf106 therapeutically could have a significant impact on several cancers. This review provides a clear direction for further studies on factors that will be affected when targeting C6orf106.

\section{Author contributions}

EK and HA conceptualized and wrote the manuscript. SB drafted and revised the manuscript. AS provided valuable suggestions during manuscript preparation and critically revised the manuscript.

\section{Ethics approval and consent to participate}

Ethical approval for this study was granted by the Institutional review board in king Fahad medical city (18379E) (July 30, 2018).

\section{Acknowledgment}

Thanks to all the peer reviewers for their opinions and suggestions.

\section{Funding}

This research received no external funding.

\section{Conflict of interest}

The authors declare no conflict of interest. 


\section{References}

[1] Tomasetti C, Li L, Vogelstein B. Stem cell divisions, somatic mutations, cancer etiology, and cancer prevention. Science. 2017; 355: 1330-1334.

[2] Meisenberg G, Simmons WH. Principles of Medical Biochemistry. 4th edn. US: Elsevier. 2016.

[3] Bray F, Ferlay J, Soerjomataram I, Siegel RL, Torre LA, Jemal A. Global cancer statistics 2018: GLOBOCAN estimates of incidence and mortality worldwide for 36 cancers in 185 countries. A Cancer Journal for Clinicians. 2018; 68: 394-424.

[4] Cree IA. Cancer biology. In Cree, IA (ed.) Cancer Cell Culture: Methods and Protocols (pp. 1-11). Totowa, NJ, USA: Humana Press. 2011.

[5] Piccart-Gebhart MJ, Procter M, Leyland-Jones B, Goldhirsch A, Untch M, Smith I, et al. Trastuzumab after adjuvant chemotherapy in her2-positive breast cancer. New England Journal of Medicine. 2005; 353: 1659-1672.

[6] Zugazagoitia J, Guedes C, Ponce S, Ferrer I, Molina-Pinelo S, Paz-Ares L. Current Challenges in Cancer Treatment. Clinical Therapeutics. 2016; 38: 1551-1566.

[7] Jiang G, Zhang X, Zhang Y, Wang L, Fan C, Xu H, et al. A novel biomarker C6orf106 promotes the malignant progression of breast cancer. Tumour Biology. 2015; 36: 7881-7889.

[8] Schiffman JD, Fisher PG, Gibbs P. Early detection of cancer: past, present, and future. American Society of Clinical Oncology educational book. 2015; 57-65.

[9] Henry NL, Hayes DF. Cancer biomarkers. Molecular Oncology. 2012; 6: 140-146.

[10] Easton DF, Ford D, Bishop DT. Breast and ovarian cancer incidence in BRCA1-mutation carriers. Breast Cancer Linkage Consortium. American Journal of Human Genetics. 1995; 56: 265271.

[11] Liu J, Zhang C, Zhao Y, Feng Z. MicroRNA Control of p53. Journal of Cellular Biochemistry. 2017; 118: 7-14.

[12] Villalobos P, Wistuba II. Lung Cancer Biomarkers. Hematology/Oncology Clinics of North America. 2017; 31: 13-29.

[13] Renaud S, Romain B, Falcoz P, Olland A, Santelmo N, Brigand $\mathrm{C}$, et al. KRAS and BRAF mutations are prognostic biomarkers in patients undergoing lung metastasectomy of colorectal cancer. British Journal of Cancer. 2015; 112: 720-728.

[14] Delgado AP, Brandao P, Chapado MJ, Hamid S, Narayanan R. Open reading frames associated with cancer in the dark matter of the human genome. Cancer Genomics \& Proteomics. 2014; 11: 201-213.

[15] Bunz F. Principles of Cancer Genetics. 2nd edn. Netherlands: Springer. 2016.

[16] Li G. Mechanisms and functions of DNA mismatch repair. Cell Research. 2008; 18: 85-98.

[17] Pal T, Permuth-Wey J, Sellers TA. A review of the clinical relevance of mismatch-repair deficiency in ovarian cancer. Cancer. 2008; 113: 733-742.

[18] Bernstein C, Bernstein H. Epigenetic reduction of DNA repair in progression to gastrointestinal cancer. World Journal of Gastrointestinal Oncology. 2015; 7: 30-46.

[19] Zhang X, Miao Y, Yu X, Zhang Y, Jiang G, Liu Y, et al. C6orf106 enhances NSCLC cell invasion by upregulating vimentin, and downregulating E-cadherin and P120ctn. Tumour Biology. 2015; 36: 5979-5985.

[20] Ambrose RL, Brice AM, Caputo AT, Alexander MR, Tribolet L, Liu YC, et al. Molecular characterisation of ILRUN, a novel inhibitor of proinflammatory and antimicrobial cytokines. Heliyon. 2020; 6: e04115.

[21] Mungall AJ, Palmer SA, Sims SK, Edwards CA, Ashurst JL, Wilming L, et al. The DNA sequence and analysis of human chromosome 6. Nature. 2003; 425: 805-811.

[22] ILRUN inflammation and lipid regulator with UBA-like and NBR1-like domains [Homo sapiens (human)]. 2021. Available at: https://www.ncbi.nlm.nih.gov/gene/64771 (Accessed: 15 March 2021).

[23] Slager SL, Camp NJ, Conde L, Shanafelt TD, Achenbach SJ, Rabe KG, et al. Common variants within 6p21.31 locus are associated with chronic lymphocytic leukaemia and, potentially, other non-Hodgkin lymphoma subtypes. British Journal of Haematology. 2012; 159: 572-576.

[24] Prat A, Cruz C, Hoadley KA, Díez O, Perou CM, Balmaña J. Molecular features of the basal-like breast cancer subtype based on BRCA1 mutation status. Breast Cancer Research and Treatment. 2014; 147: 185-191.

[25] Alluri P, Newman LA. Basal-Like and Triple-Negative Breast Cancers: searching for positives among many negatives. Surgical Oncology Clinics of North America. 2014; 23: 567-577.

[26] Thul PJ, Åkesson L, Wiking M, Mahdessian D, Geladaki A, Ait Blal H, et al. A subcellular map of the human proteome. Science. 2017; 356: eaal3321.

[27] Lonsdale J, Thomas J, Salvatore M, Phillips R, Lo E, Shad S, et al. The Genotype-Tissue Expression (GTEx) project. Nature Genetics. 2013; 45: 580-585.

[28] Uhlén M, Fagerberg L, Hallström BM, Lindskog C, Oksvold P, Mardinoglu A, et al. Proteomics. Tissue-based map of the human proteome. Science. 2015; 347: 1260419.

[29] Uechi H, Kuranaga E, Iriki T, Takano K, Hirayama S, Miura M, et al. Ubiquitin-Binding Protein CG5445 Suppresses Aggregation and Cytotoxicity of Amyotrophic Lateral Sclerosis-Linked TDP-43 in Drosophila. Molecular and Cellular Biology. 2018; 38: e00195-e00117.

[30] Li X, Dong M, Zhou J, Zhu D, Zhao J, Sheng W. C6orf106 accelerates pancreatic cancer cell invasion and proliferation via activating ERK signaling pathway. Molecular and Cellular Biochemistry. 2019; 454: 87-95.

[31] Ambrose RL, Liu YC, Adams TE, Bean AGD, Stewart CR C6orf106 is a novel inhibitor of the interferon-regulatory factor 3-dependent innate antiviral response. Journal of Biological Chemistry. 2018; 293: 10561-10573.

[32] McCain J. The MAPK (ERK) Pathway: Investigational Combinations for the Treatment of BRAF-Mutated Metastatic Melanoma. A Peer-Reviewed Journal for Formulary Management. 2013; 38: 96-108.

[33] Oláh J, Vincze O, Virók D, Simon D, Bozsó Z, Tõkési N, et al. Interactions of pathological hallmark proteins: tubulin polymerization promoting protein/p25, beta-amyloid, and alphasynuclein. Journal of Biological Chemistry. 2011; 286: 34088 34100.

[34] Hansel DE, Rahman A, Wehner S, Herzog V, Yeo CJ, Maitra A. Increased expression and processing of the Alzheimer amyloid precursor protein in pancreatic cancer may influence cellular proliferation. Cancer Research. 2003; 63: 7032-7037.

[35] Lim S, Yoo BK, Kim H, Gilmore HL, Lee Y, Lee H, et al. Amyloid- $\beta$ precursor protein promotes cell proliferation and motility of advanced breast cancer. BMC Cancer. 2014; 14: 928

[36] Meng JY, Kataoka H, Itoh H, Koono M. Amyloid beta protein precursor is involved in the growth of human colon carcinoma cell in vitro and in vivo. International Journal of Cancer. 2001; 92: 31-39.

[37] Pandey P, Sliker B, Peters HL, Tuli A, Herskovitz J, Smits K, et al. Amyloid precursor protein and amyloid precursor-like protein 2 in cancer. Oncotarget. 2016; 7: 19430-19444.

[38] Abdelmohsen K, Srikantan S, Yang X, Lal A, Kim HH, Kuwano $\mathrm{Y}$, et al. Ubiquitin-mediated proteolysis of HuR by heat shock. EMBO Journal. 2009; 28: 1271-1282.

[39] Tran H, Maurer F, Nagamine Y. Stabilization of Urokinase and Urokinase Receptor mRNAs by HuR is Linked to its Cytoplasmic Accumulation Induced by Activated Mitogen-Activated Protein Kinase-Activated Protein Kinase 2. Molecular and Cellular Biology. 2003; 23: 7177-7188. 
[40] Lukosiute-Urboniene A, Jasukaitiene A, Silkuniene G, Barauskas V, Gulbinas A, Dambrauskas Z. Human antigen R mediated post-transcriptional regulation of inhibitors of apoptosis proteins in pancreatic cancer. World Journal of Gastroenterology. 2019; 25: 205-219.

[41] Wang J, Guo Y, Chu H, Guan Y, Bi J, Wang B. Multiple functions of the RNA-binding protein HuR in cancer progression, treatment responses and prognosis. International Journal of Molecular Medicine. 2013; 14: 10015-10041.

[42] Jiang G, Zhang X, Wang L, Lin X, Yu J, Wang E, et al. FBXO25 promotes cell proliferation, invasion, and migration of NSCLC. Tumor Biology. 2016; 37: 14311-14319.

[43] Baumann U, Fernández-Sáiz V, Rudelius M, Lemeer S, Rad R, Knorn A, et al. Disruption of the PRKCD-FBXO25-HAX-1 axis attenuates the apoptotic response and drives lymphomagenesis. Nature Medicine. 2014; 20: 1401-1409.

[44] Huttlin EL, Bruckner RJ, Paulo JA, Cannon JR, Ting L, Baltier $\mathrm{K}$, et al. Architecture of the human interactome defines protein communities and disease networks. Nature. 2017; 545: 505509.

[45] Sherman M, Multhoff G. Heat shock proteins in cancer. Annals of the New York Academy of Sciences. 2007; 1113: 192-201.

[46] Shan N, Zhou W, Zhang S, Zhang Y. Identification of HSPA8 as a candidate biomarker for endometrial carcinoma by using iTRAQ-based proteomic analysis. OncoTargets and Therapy. 2016; 9: 2169-2179.

[47] Tanimura S, Hirano A, Hashizume J, Yasunaga M, Kawabata $\mathrm{T}$, Ozaki K, et al. Anticancer drugs up-regulate HspBP1 and thereby antagonize the prosurvival function of Hsp70 in tumor cells. Journal of Biological Chemistry. 2007; 282: 3543035439.

[48] Uhlen M, Zhang C, Lee S, Sjöstedt E, Fagerberg L, Bidkhori $\mathrm{G}$, et al. A pathology atlas of the human cancer transcriptome. Science. 2017; 357: eaan2507.

[49] Lodish H, Berk A, Zipursky SL, et al. Molecular Cell Biology. 4th edition. New York: W. H. Freeman. 2000.

[50] Charlaftis N, Suddason T, Wu X, Anwar S, Karin M, Gallagher E. The MEKK1 PHD ubiquitinates TAB1 to activate MAPKs in response to cytokines. EMBO Journal. 2014; 33: 2581-2596.

[51] Schlesinger TK, Bonvin C, Jarpe MB, Fanger GR, Cardinaux J, Johnson GL, et al. Apoptosis stimulated by the $91-\mathrm{kDa}$ caspase cleavage MEKK1 fragment requires translocation to soluble cellular compartments. Journal of Biological Chemistry. 2002; 277: 10283-10291.

[52] Warr N, Bogani D, Siggers P, Brixey R, Tateossian H, Dopplapudi A, et al. Minor abnormalities of testis development in mice lacking the gene encoding the MAPK signalling component, MAP3K1. PLoS ONE. 2011; 6: e19572.

[53] Cuevas BD, Winter-Vann AM, Johnson NL, Johnson GL. MEKK1 controls matrix degradation and tumor cell dissemination during metastasis of polyoma middle-T driven mammary cancer. Oncogene. 2006; 25: 4998-5010.

[54] Su F, Li H, Yan C, Jia B, Zhang Y, Chen X. Depleting MEKK1 expression inhibits the ability of invasion and migration of human pancreatic cancer cells. Journal of Cancer Research and Clinical Oncology. 2009; 135: 1655-1663.

[55] Andrews PS, Schneider S, Yang E, Michaels M, Chen H, Tang $\mathrm{J}$, et al. Identification of substrates of SMURF1 ubiquitin ligase activity utilizing protein microarrays. Assay and Drug Development Technologies. 2010; 8: 471-487.

[56] David D, Nair SA, Pillai MR. Smurf E3 ubiquitin ligases at the cross roads of oncogenesis and tumor suppression. Biochimica Et Biophysica Acta. 2013; 1835: 119-128.

[57] Heikel G, Choudhury NR, Michlewski G. The role of Trim25 in development, disease and RNA metabolism. Biochemical Society Transactions. 2016; 44: 1045-1050.

[58] Versteeg GA, Benke S, García-Sastre A, Rajsbaum R. InTRIMsic immunity: Positive and negative regulation of immune signaling by tripartite motif proteins. Cytokine \& Growth Factor
Reviews. 2014; 25: 563-576.

[59] Qin Y, Cui H, Zhang H. Overexpression of TRIM25 in Lung Cancer Regulates Tumor Cell Progression. Technology in Cancer Research \& Treatment. 2016; 15: 707-715.

[60] Zhang P, Elabd S, Hammer S, Solozobova V, Yan H, Bartel F, et al. TRIM25 has a dual function in the p53/Mdm2 circuit. Oncogene. 2015; 34: 5729-5738.

[61] Choudhury NR, Heikel G, Trubitsyna M, Kubik P, Nowak JS, Webb S, et al. RNA-binding activity of TRIM 25 is mediated by its PRY/SPRY domain and is required for ubiquitination. BMC Biology. 2017; 15: 105.

[62] Nelson J, McFerran NV, Pivato G, Chambers E, Doherty C, Steele D, et al. The $67 \mathrm{kDa}$ laminin receptor: structure, function and role in disease. Bioscience Reports. 2008; 28: 33-48.

[63] Givant-Horwitz V, Davidson B, Reich R. Laminin-induced signaling in tumor cells. Cancer Letters. 2005; 223: 1-10.

[64] Wang J, Huo K, Ma L, Tang L, Li D, Huang X, et al. Toward an understanding of the protein interaction network of the human liver. Molecular Systems Biology. 2017; 13: 965.

[65] Neggers JE, Vercruysse T, Jacquemyn M, Vanstreels E, Baloglu $\mathrm{E}$, Shacham S, et al. Identifying drug-target selectivity of smallmolecule CRM1/XPO1 inhibitors by CRISPR/Cas9 genome editing. Chemistry \& Biology. 2015; 22: 107-116.

[66] Kırlı K, Karaca S, Dehne HJ, Samwer M, Pan KT, Lenz C, et al. A deep proteomics perspective on CRM1-mediated nuclear export and nucleocytoplasmic partitioning. ELife. 2015; 4: e11466.

[67] Hochegger H, Takeda S, Hunt T. Cyclin-dependent kinases and cell-cycle transitions: does one fit all? Nature Reviews Molecular Cell Biology. 2008; 9: 910-916.

[68] Kanakkanthara A, Jeganathan KB, Limzerwala JF, Baker DJ, Hamada M, Nam H, et al. Cyclin a2 is an RNA binding protein that controls Mre11 mRNA translation. Science. 2016; 353: 1549-1552.

[69] Akimov IA, Chernolovskaia EL. The CCNB1, HER2, and PKC silencing induced by small interfering RNA decreases a division of different human cancer cells with interfering RNA decreases a different efficiency. Molecular Biology. 2010; 44: 98-106. (In Russian)

[70] Pines J, Hunter T. Cyclins a and B1 in the human cell cycle. Ciba Foundation Symposium. 1992; 170: 187-204.

[71] Gomez LA, de Las Pozas A, Reiner T, Burnstein K, Perez-Stable C. Increased expression of cyclin B1 sensitizes prostate cancer cells to apoptosis induced by chemotherapy. Molecular Cancer Therapeutics. 2007; 6: 1534-1543.

[72] Gabay M, Li Y, Felsher DW. MYC activation is a hallmark of cancer initiation and maintenance. Cold Spring Harbor Perspectives in Medicine. 2014; 4: a014241.

[73] Miller DM, Thomas SD, Islam A, Muench D, Sedoris K. CMyc and cancer metabolism. Clinical Cancer Research. 2012; 18: 5546-5553.

[74] van Roy F. Beyond E-cadherin: roles of other cadherin superfamily members in cancer. Nature Reviews Cancer. 2014; 14: 121-134.

[75] Tadokoro A, Kanaji N, Liu D, Yokomise H, Haba R, Ishii T, et al. Vimentin Regulates Invasiveness and is a Poor Prognostic Marker in Non-small Cell Lung Cancer. Anticancer Research. 2016; 36: 1545-1551.

[76] Kidd ME, Shumaker DK, Ridge KM. The role of vimentin intermediate filaments in the progression of lung cancer. American Journal of Respiratory Cell and Molecular Biology. 2014; 50: $1-6$.

[77] Lamouille S, Xu J, Derynck R. Molecular mechanisms of epithelial-mesenchymal transition. Nature Reviews Molecular Cell Biology. 2014; 15: 178-196.

[78] Kourtidis A, Ngok SP, Anastasiadis PZ. P120 catenin: an essential regulator of cadherin stability, adhesion-induced signaling, and cancer progression. Progress in Molecular Biology and Translational Science. 2013; 116: 409-432. 
[79] Bi X, Kuwano T, Lee PC, Millar JS, Li L, Shen Y, et al. ILRUN, a Human Plasma Lipid GWAS Locus, Regulates Lipoprotein Metabolism in Mice. Circulation Research. 2020; 127: 13471361.

Abbreviations: C6orf106, Chromosome 6 open reading frame 106; HER2, Human epidermal growth factor receptor 2; KRAS, Kirsten rat sarcoma viral oncogene homolog; NSCLC, Non-small cell lung cancer; ORF, Open reading frame; MMR, Mismatch repair; ALS, Amyotrophic lateral sclerosis; TNBC, Triple-negative breast cancer; PR, Progesterone hormone receptor; ER, Estrogen hormone receptor; HER2, Human epidermal growth factor receptor 2; APP, Amyloid-beta precursor protein; RBPs, RNA-binding proteins; MCL, Mantle Cell Lymphoma; N-cadherin, neuronal cadherin; EMT, epithelial-to-mesenchymal transition.
Keywords: Cancer; Biomarker; Chromosome 6 open reading frame 106; Gene expression

Send correspondence to: Saeed Baradwan, Department of Obstetrics and Gynecology, King Faisal Specialist Hospital and Research Centre, 11564 Jeddah, Saudi Arabia, E-mail: dr.saeed_bardwan@yahoo.com 